\title{
Optical investigation of a wavy ageing interface
}

\author{
C. Picard*, L. Davoust \\ LEGI, BP 53, 38041 Grenoble Cedex 9, France
}

\begin{abstract}
As an attempt to develop a sensitive device for biomolecule detection, a micromechanical methodology, based on the rheological change of an air/water interface is put forward [1]. Capillary waves induced from the vertical electrodynamic vibration of a brimfull cylindrical tank, filled with water, stand as a good way to identify dilational elasticity and viscosity of an ageing interface. Of prior interest for us, the complex wave number and the frequency of the waves are obtained through an optical technique, which allow us to recognise accurately the whole interface geometry. These two parameters and a modeling based on a dispersion relation are jointly used to identify the dilational rheology of the interface [2]. The validity of the methodology is assessed making use of palmitic acid as surfactant.
\end{abstract}

Keywords: Wavy ageing interface; Optical investigation; Palmitic acid

\section{Introduction}

Detection and characterization of biological material is currently identified as a priority in biotechnology. Up to now most attention has been focused on biochips [3]. In this paper, we present a drastically different technique based on the rheological properties of a functionalized air/water interface to which biomolecules adsorb [1]. As a typical example, it is worth evoking the hybridization of solubilized target-DNAstrands on receptor-DNA-strands initially adsorbed to a lipidic monolayer spread over an air/water interface. In that configuration, the surface pressure of the interface is proved to be sensitive to the hybridization process [4]. This paper aims at developing a rheological diagnostic based on the elongation of a functionalized air/water interface and which is expected to be more sensitive than a surface pressure measurement, alone.

Surface rheology measurements are usually carried out thanks to intrusive techniques, such as Stokes drag based devices [5] or Taylor-Couette viscometer for shearing parameters determination, while pulsed drops [6] or traveling waves systems are regularly used for dilational elasticity measure-

\footnotetext{
* Corresponding author. Tel.: +334565286 08; fax: +33476827022. E-mail address: cyril.picard@hmg.inpg.fr (C. Picard).
}

ment. Unlike these widespread methods, which cannot fulfill most of biological requirements, a non-intrusive technique, based on small amounts of reactants and preventing from any external contamination, is proposed.

A small cylindrical tank filled with water is vertically shaked. The standing cylindrical capillary waves so-excited are responsible for the axi-symmetrical deformation of the air/water interface which can be functionalized. The geometry of the deformed interface is able to change as target molecules adsorb at the bio-functionalized air/water interface. Tow different optical techniques are developed to characterise the geometry modification. A refractometrybased method allow us to rebuilt precisely the whole interface geometry whereas an interferometry-based method is developed to monitor continuously the surface elevation at the center of the tank, where the surface remains horizontal during its vertical motion. The first technique operates only for very slow geometry evolution, whereas the second one can detect fast changes of the surface elevation. Therefore, both techniques are complementary. Interferometry is convenient for concrete applications such as bio-molecule detection, but not sufficient for fundamental studies such as rheology identification, which needs an accurate recognition of the interface geometry carried out with refractometry. The interferometry method, not described here, will be the focus of another paper. 
Considering waves, whose amplitude is sufficiently small, the liquid motion is described by the linearized NavierStokes equations. Wave number and damping coefficient appearing in the solution of the hydrodynamics equations are experimentally determined from the proposed setup. The normal component of momentum balance at the interface and the dispersion relation for cylindrical standing waves are then jointly used to identify the surface tension and the elasticity modulus without any assumption on the dilational viscosity value.

\section{Hydrodynamic theory}

\subsection{Nearly standing waves}

We consider the limiting case of a free surface between a gas phase and an aqueous phase, contained in a cylindrical tank. The interface, limited by the $R$ radius circular tank pin-edge, is deformed by nearly standing ripples generated by vibrating the tank in the vertical direction $z$. The liquid fills the half-space $z<0$. The interface at rest corresponds to the plane $z=0$ and the pressure acting upon it from both phases is the ambient pressure $p_{0}$. Considering an axi-symmetrical deformation, the velocity and the pressure of the liquid depend only on the radial $r$ and vertical $z$ coordinates. Solution of the linearized Navier-Stokes equations for propagating cylindrical waves is largely described in the literature $([2,7,8])$. As mentioned by Saylor et al. [9], so-supplied nearly standing waves can be seen as the superimposition of damped inward-traveling waves and damped outward traveling waves whose amplitude is the same at $r=0$. Therefore, solution for nearly standing ripples is easily established following the analytical development by Bock [7] for damped traveling ripples. We consider the velocity components and the pressure, written, according to the Helmotz decomposition, in terms of the potential $\varphi$ and stream $\psi$ functions:

$v_{r}=-\frac{\partial \varphi}{\partial r}-\frac{\partial \psi}{\partial z}$

$v_{z}=-\frac{\partial \varphi}{\partial z}+\frac{1}{r} \frac{\partial(r \psi)}{\partial r}$

$p=p_{0}-\rho g z+\rho \frac{\partial \varphi}{\partial t}$

where $\rho$ is the water density and $g$ the gravity acceleration. The potential and stream functions, characterising the nearly standing ripples of interest, can be expressed as:

$\varphi=A J_{0}(k r) \mathrm{e}^{k z} \mathrm{e}^{i \omega t}$

$\psi=i B J_{1}(k r) \mathrm{e}^{m z} \mathrm{e}^{i \omega t}$,

where, $J_{0}$ and $J_{1}$ are the first-kind Bessel function of the zeroth order and of the first order, respectively. These functions are the sum of Hankel functions of the first kind and second kind used respectively to describe outward-traveling and inward-traveling waves:

$J_{n}(k r)=H_{n}^{(1)}(k r)+H_{n}^{(2)}(k r)$.

Let $\kappa$ and $\beta$ represent the wave number and the damping coefficient. The complex wave number $k$ and the number $m$ are defined by:

$k=\kappa+i \beta$,

$m^{2}=k^{2}+\frac{i \omega \rho}{\mu} \quad$ and $\quad \Re e(m)>0$.

Since the velocity components at the surface, $v_{r s}$ and $v_{z s}$ are defined as Lagrangian derivatives of radial displacement $\xi$ and vertical displacement $\zeta$ of a surface particle, one obtains:

$v_{r s}=J_{1}(k r) \mathrm{e}^{i \omega t}\left(k A \mathrm{e}^{k z}-i m B \mathrm{e}^{m z}\right)=\frac{\partial \xi}{\partial t}$,

$v_{z s}=J_{0}(k r) \mathrm{e}^{i \omega t}\left(i k B \mathrm{e}^{m z}-k A \mathrm{e}^{k z}\right) \simeq \frac{\partial \zeta}{\partial t}$.

Consequently the radial and vertical displacements of the surface are:

$\xi=-\frac{i k A+m B}{\omega} J_{1}(k r) \mathrm{e}^{i \omega t}$,

$\zeta=\frac{k B-i k A}{\omega} J_{0}(k r) \mathrm{e}^{i \omega t}$

The pressure at the surface writes therefore:

$p_{s}=p_{0}-\left(\frac{\rho g}{\omega}(k B-i k A)+i \rho \omega A\right) J_{0}(k r) \mathrm{e}^{i \omega t}$.

\subsection{Surface rheology characterization}

\subsubsection{Momentum balance and dispersion relation}

An interfacial infinitesimal area is submitted to stresses from the liquid and stresses intrinsic to the interface if gaz, located above, is seen as a neutral phase. Hence the normal component of the momentum balance, at the interface, writes as:

$\frac{\sigma}{r} \frac{\partial}{\partial r}\left[r \frac{\partial \zeta}{\partial r}\right]=p_{0}-p+\left.2 \mu \frac{\partial v_{z}}{\partial z}\right|_{z=0}$.

And the tangential component of the momentum balance tangential at the interface writes as:

$\varepsilon \frac{\partial}{\partial r}\left[\frac{1}{r} \frac{\partial(r \xi)}{\partial r}\right]=\mu\left(\left.\frac{\partial v_{r}}{\partial z}\right|_{z=0}+\left.\frac{\partial v_{z}}{\partial r}\right|_{z=0}\right)$.

Here, $\mu$ is the water viscosity, $\sigma$ the surface tension of the interface, and $\epsilon=\epsilon_{\mathrm{d}}+\mathrm{i} \omega \eta_{\mathrm{d}}$ is the surface elasticity modulus with $\epsilon_{\mathrm{d}}$ and $\eta_{\mathrm{d}}$ which are referred to as the dilational elasticity and the dilational viscosity. If we substitute the relations (9), (10) and (13) in (14) and (15) and make use of recursion relations between Bessel functions and their derivatives, we find the same relations as Lucassen-Reynders and Lucassen $[2,7]$ : for the normal stresses, 
$i A\left(\sigma-\frac{\rho \omega^{2}}{k^{3}}+2 i \mu \frac{\omega}{k}+\frac{\rho g}{k^{2}}\right)+B\left(\sigma+2 i \mu \frac{\omega m}{k^{2}}+\frac{\rho g}{k^{2}}\right)=0$,

and for the tangential stresses.

$A(k \varepsilon+2 i \mu \omega)-i B\left(m \varepsilon+i \mu \omega \frac{k^{2}+m^{2}}{k^{2}}\right)=0$,

The dispersion relation, obtained from (16) and (17) by equating the determinant of the coefficients of $A$ and $B$ to zero, is proposed with a similar form as the one chosen in [2]:

$$
\begin{aligned}
& \left(\varepsilon+i \mu \omega \frac{k+m}{k^{2}}\right)\left(\frac{\sigma k^{2}}{\omega}+i \mu(k+m)+\frac{\rho g}{\omega}-\frac{\rho \omega}{k}\right) \\
& =-\omega \mu^{2}\left(1-\frac{m}{k}\right)^{2} .
\end{aligned}
$$

\subsubsection{Surface tension and elasticity modulus}

Define the complex number $s_{A}$ and $s_{B}$ by:

$s_{A}=-\frac{\rho \omega^{2}}{k^{3}}+2 i \mu \frac{\omega}{k}+\frac{\rho g}{k^{2}}$,

$s_{B}=2 i \mu \frac{\omega m}{k^{2}}+\frac{\rho g}{k^{2}}$.

Using these notations, the normal component of the stress balance at the interface (16) is:

$i A\left(\sigma+s_{A}\right)+B\left(\sigma+s_{B}\right)=0$.

Separating the real part and the imaginary part of this equation, one obtains the following arithmetic equations:

$B\left(\sigma+\Re e\left(s_{B}\right)\right)-A \Im m\left(s_{A}\right)=0$,
$B \Im m\left(s_{B}\right)+A\left(\sigma+\Re e\left(s_{A}\right)\right)=0$.

A non-trivial solution can be found to the system formed by (22) and (23), if the determinant formed by the coefficients of $A$ and $B$ is equated to 0 . If we make use of the following notation:

$x_{X} \equiv \Re e\left(s_{X}\right), \quad y_{X} \equiv \Im m\left(s_{X}\right)$,

the determinant is:

$\sigma^{2}+\left(x_{B}+x_{A}\right) \sigma+x_{A} x_{B}+y_{A} y_{B}=0$,

from which one selects the physical roots:

$\sigma=\frac{1}{2}\left(\sqrt{\left(x_{B}-x_{A}\right)^{2}-4 y_{B} y_{A}}-x_{B}-x_{A}\right)$.

This relation is one of the main result of this paper. One can show easily that when assessing the value of $\sigma$ in this way, the capillary waves are equivalently handled with the point of view of a potential viscous wavy flow.
Thus, once $k$ and $\omega$ are known, it is possible to determine the surface tension $\sigma$, which is next substituted into the dispersion relation (18) so as to obtain the elasticity modulus $\epsilon$ :

$\varepsilon=\frac{\omega^{2} \mu^{2}(k-m)^{2}}{\rho \omega^{2} k-i \mu k^{2}(k+m)-\rho g k^{2}-\sigma k^{4}-i \mu \omega \frac{k+m}{k^{2}}}$.

In this way, neither supplementary assumption on dilational viscosity value nor tedious minimization techniques are needed to identify $\sigma$ and $\epsilon$.

\subsection{Determination of the complex wave number}

\subsubsection{Approximation $\beta R \ll 1$}

Waves are not standing but nearly standing, because of the damping effect which implies that $k$ is a complex wave number. This means, as mentioned by Saylor et al. [9], that waves behave more and more like inward-traveling waves when $r$ increases and tends to the outer radius of the water bath. The only point where the wave is truly a standing wave is the center of the interface. There, the surface remains horizontal during its vertical displacement. Assuming that $\beta R$ is sufficiently small, a very good approximation of the surface elevation can be proposed in order to simplify the determination of the wave number $\kappa$ and the damping coefficient $\beta$. Using the following notations:

$\zeta=\left|\frac{k B-i k A}{\omega}\right|$,

$\phi=\arg \left(\frac{k B-i k A}{\omega}\right)$,

the elevation of the surface given by (12) can be written:

$\zeta=\zeta J_{0}(k r) \mathrm{e}^{i \omega t+\phi}$

Using the original definition of the $J_{0}$-Bessel function, one obtains:

$$
\begin{aligned}
J_{0}((\kappa+i \beta) r)= & \frac{1}{\pi} \quad{ }_{0}^{\pi} \cos (\kappa r \sin \theta) \operatorname{ch}(\beta r \sin \theta) \\
& -i \sin (\kappa r \sin \theta) \operatorname{sh}(\beta r \sin \theta) \mathrm{d} \theta
\end{aligned}
$$

Assuming $\beta R \ll 1$ one finds:

$$
\begin{aligned}
J_{0}((\kappa+i \beta) r) \simeq & \frac{1}{\pi}{ }_{0}^{\pi} \cos (\kappa r \sin \theta)-i \beta r \sin \theta \\
& \sin (\kappa r \sin \theta) \mathrm{d} \theta=J_{0}(\kappa r)-i \beta r J_{1}(\kappa r),
\end{aligned}
$$

and the approximate surface elevation found to be:

$\zeta=\zeta_{0}\left(J_{0}(\kappa r)-i \beta r J_{1}(\kappa r)\right) \mathrm{e}^{i \omega t+\phi}$. 
Consequently, the slope of the surface, which stands as the quantity of interest in this paper, writes:

$\frac{\partial \zeta}{\partial r}=-\zeta_{0} \kappa\left(J_{1}(\kappa r)+i \beta r J_{0}(\kappa r)\right) \mathrm{e}^{i \omega t+\phi}$.

\subsubsection{Identification process}

Our experiments are based on the same idea as the one proposed by Saylor et al. [9]: the measurement of the wave slope amplitude according to the radius. However, the improvement proposed in this paper is based on the fact that our measurements are not carried out along a short distance away from the center but along a complete diameter of the interface, $5 \mathrm{~cm}$ in length. The wave slope is thus measured with greater accuracy, at the vicinity of the center where its amplitude is maximum and where disturbances are minimized.

The wave slope envelope, making use of the approximation $\beta R \ll 1$, verifies:

$\left|\frac{\partial \zeta}{\partial r}\right|^{2}=\zeta_{0}^{2} \kappa^{2}\left(J_{1}^{2}(\kappa r)+\beta^{2} r^{2} J_{0}^{2}(\kappa r)\right)$.

The experimental wave slope envelope is then used to determine the parameters $\kappa$ and $\beta$. Assuming that the radial coordinate of the minima of the envelope multiply by $\kappa$ equal the roots of $J_{1}$, we first determine the value of $\kappa$ which leads to the closest match between the experimental minima and the roots of $J_{1}$, using a least-square method. Once $\kappa$ has been obtained, the square of the slope envelop can be written:

$\left|\frac{\partial \zeta}{\partial r}\right|^{2}=\zeta_{0}^{2} X(r)+\zeta_{0}^{2} \beta^{2} Y(r)$

with $X$ and $Y$ two known functions of the radial coordinate. The parameter $\zeta_{0}$ and $\beta$ are calculated using again a leastsquare minimization technique to fit the experimental points with the theoretical relation (35). One points out that the value of $\beta$ is independent of the amplitude of the experimental signal. Consequently, even if the gain of the device used to obtain the wave slope is unknown, $\beta$ can be calculated, assuming the gain is constant. Substituting $\zeta_{0}, \kappa$ and $\beta$, obtained with this simple method, in the theoretical envelop (34), leads to a very good agreement with the experimental data's.

\section{Experimental setup}

\subsection{Vibrating reactor}

The experiments are carried out using a cylindrical glass tank whose outer diameter is $5 \mathrm{~cm}$. It is mounted at the center of a circular plexiglass plate supported by height springs at its periphery. Three small solenoids are symmetrically attached to the plate around the container. A home-made radial-field magnet is adjusted around each solenoid. Three amplifiers supply the solenoids. The three of them are supplied with the same sinusoidal signal but the gain of each amplifier can be adjusted independently of the others so that the container is shaked exactly axi-symmetrically. The horizontality of the whole apparatus can be adjusted so that the container pinedge is exactly horizontal. The vibration frequency is tuned between 10 and $100 \mathrm{~Hz}$ (Fig. 1).

\subsection{Scanning device}

A HeNe laser beam initially horizontal is driven to the interface through a mirror placed under the container. This mirror is fixed on a motorized support which permits the motion of the mirror along a diameter of the tank. Therefore, it is possible to scan the interface with the laser beam at any

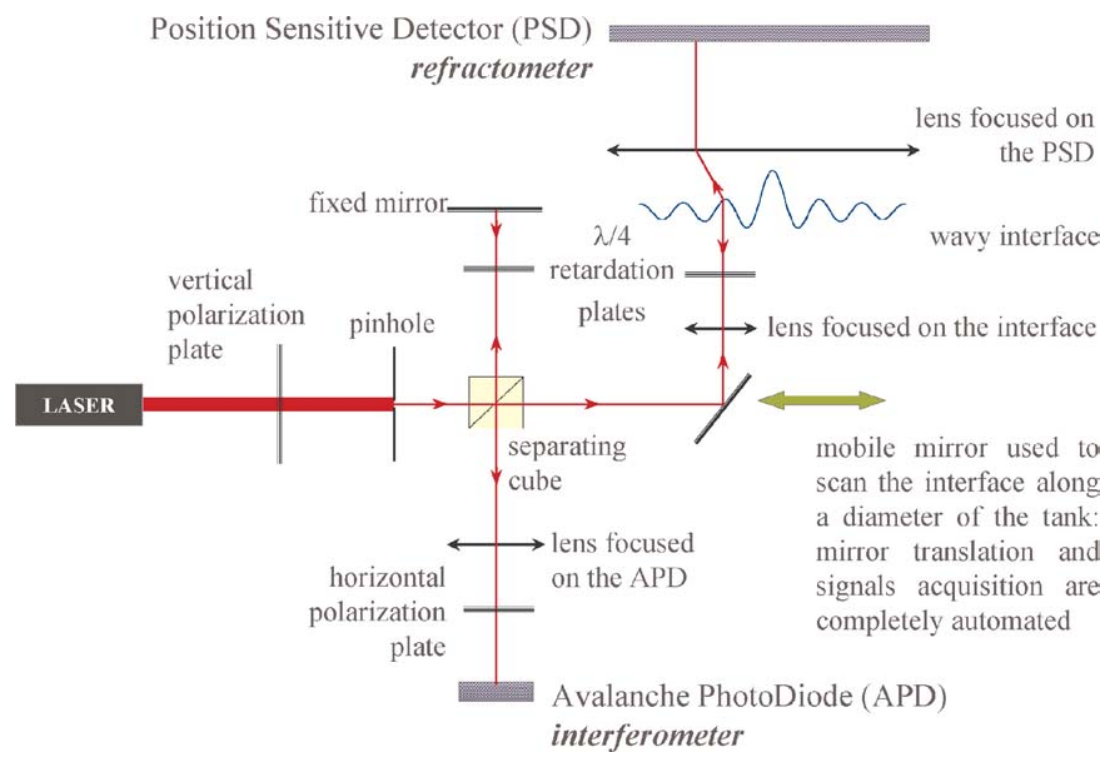

Fig. 1. Schematic diagram of the experimental setup. 
location along a diameter of the tank. A lens, also mounted on the mobile support and placed between the mirror and the container bottom, is used to focus conveniently the laser beam onto the interface in order to obtain the best spatial resolution even for very small capillary wave length.

\subsection{Refractometry}

A one-dimensional Position Sensitive Detector (PSD) is mounted above the tank and aligned along with the mirror motion direction. A lens is placed between the air/water interface and the PSD at a distance equal to its focus length so that whatever the location of the laser beam at the interface, the refracted part of the laser beam will impact the PSD at a location which depends only on the interface slope.

\subsection{Automation}

An automation program has been developed to control jointly the displacement of the motorized support and the
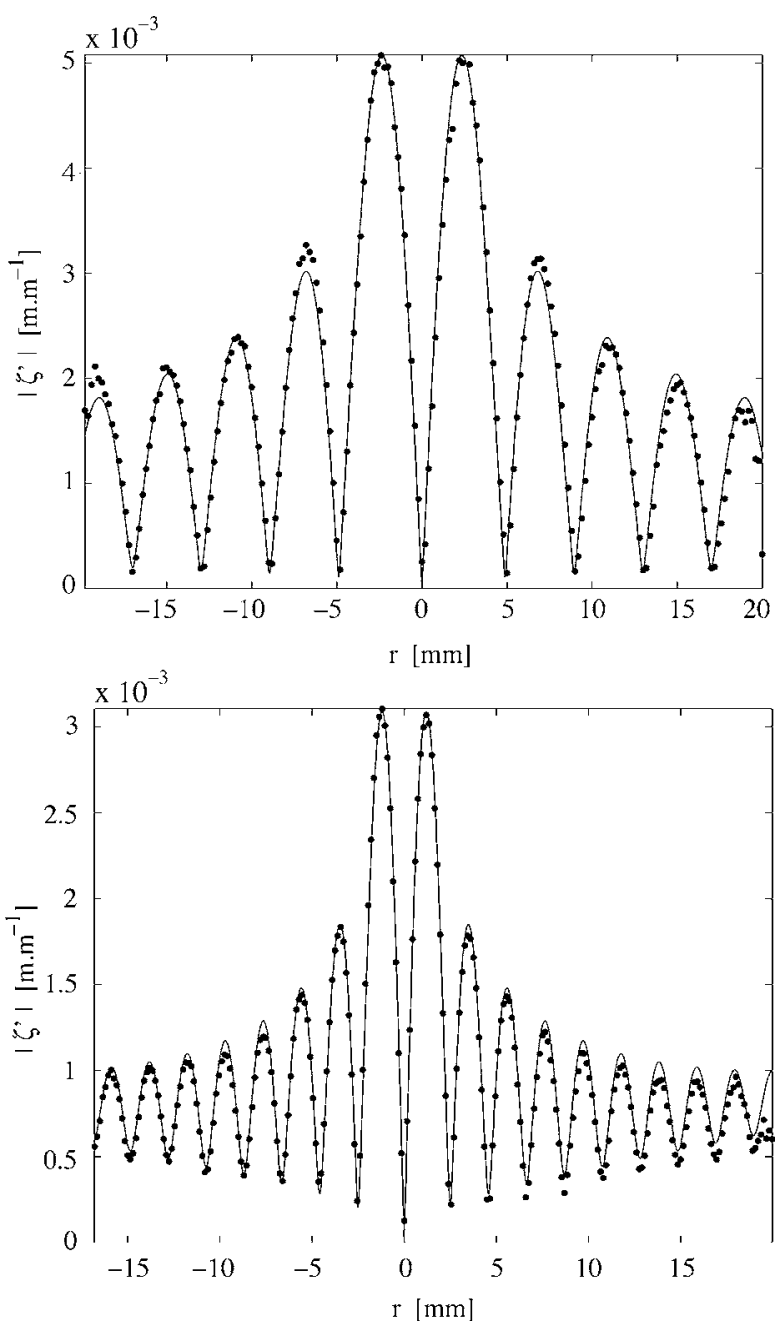

Fig. 2. Wave slope amplitude against radius for pure water surface shaked at $32 \mathrm{~Hz}$ (first figure) and at $79 \mathrm{~Hz}$ (second figure), dots are measurements and lines model fit.

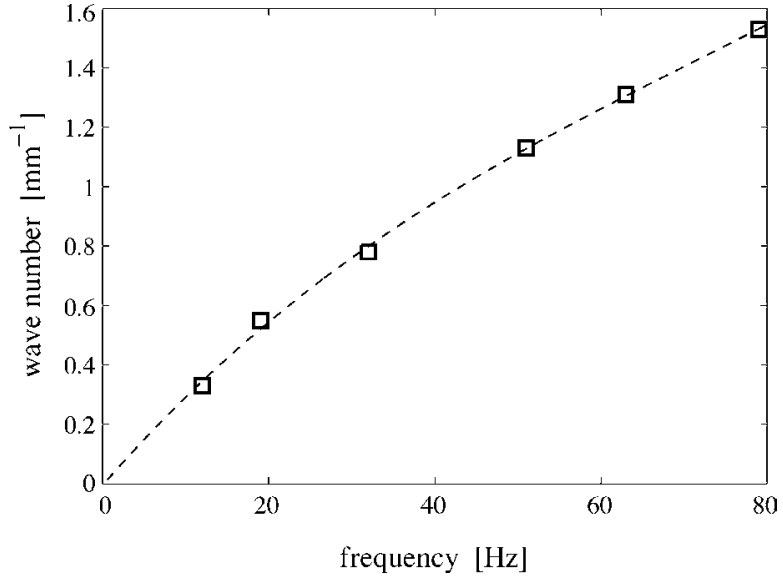

Fig. 3. Evolution of the wave number vs. frequency in the case of a pure water surface.

PSD signal acquisition so that as many locations as necessary along the radius can be characterized.

\section{Validation of the method}

The validation stage presented here has been carried out in order to exhibit the accuracy of the recognition process of the surface slope and to point out the sensitivity to surface contamination. The influence of the frequency is first investigated in the case of a pure water surface. Then, the evolution of the deformation of a surface contaminated by palmitic acid is studied when varying the surface concentration at a constant frequency. The sensitivity of the dilational rheology according to surface concentration is also characterized. The whole of the measurement have been conducted at $23^{\circ} \mathrm{C}$.

Fig. 2 presents two typical plots of the wave slope envelope according to the radius position for a pure water surface shaked at two different frequencies. Similarly to the curve by

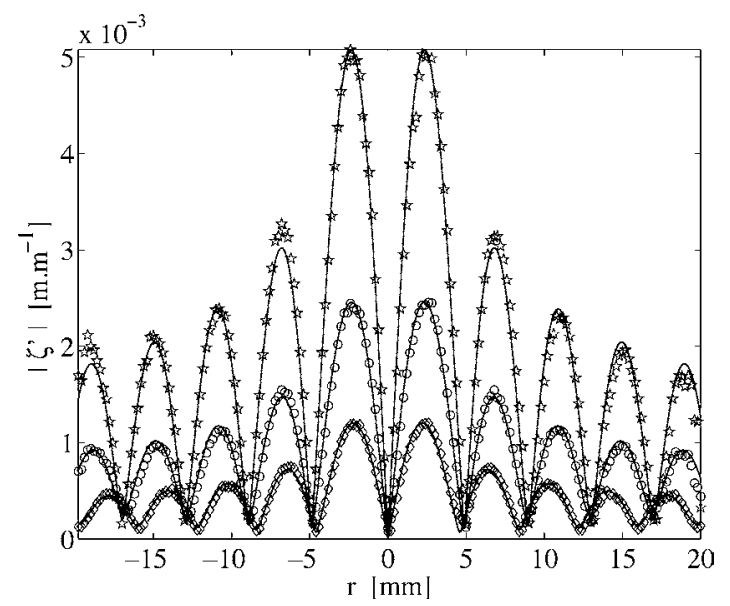

Fig. 4. Wave slope amplitude against radius at $32 \mathrm{~Hz}$ when surface concentration of palmitic acid increases, markers are experimental measurements and lines are the model fit: ( $(5)$ pure water, ( $(\circ) \Gamma=4.6 \times 10^{-6} \mathrm{~mol} \mathrm{~m}^{-2}$, $(\diamond) \Gamma=6.9 \times 10^{-6} \mathrm{~mol} \mathrm{~m}^{-2}$. 


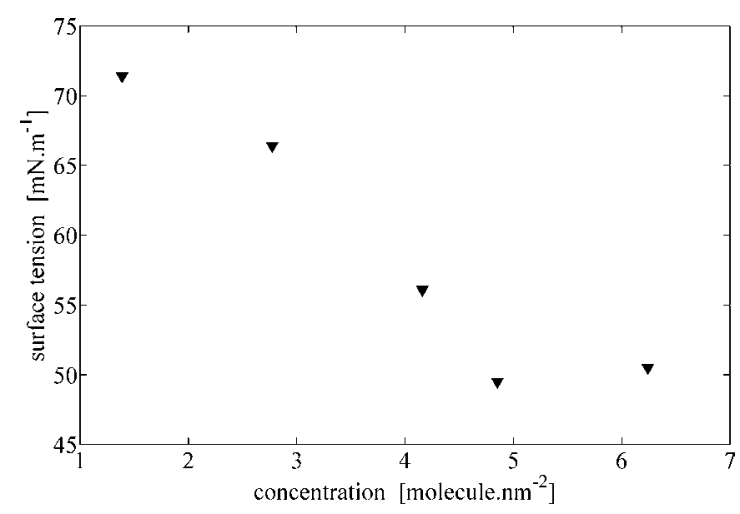

Fig. 5. Evolution of the surface tension vs. surface concentration of palmitic acid.

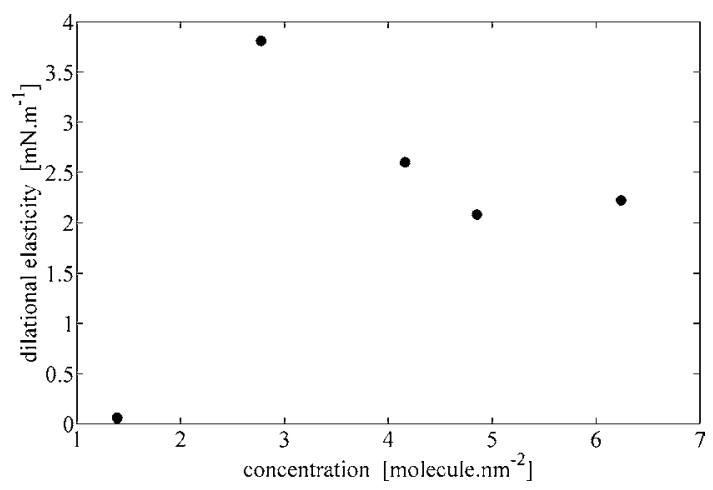

Fig. 6. Evolution of the dilational elasticity vs. surface concentration of palmitic acid.

Saylor et al. [9], each point is the amplitude of a sinusoidal time trace measured at the considered radius. In order to verify that the deformation is truly symmetrical, the surface is scanned from negative to positive values of the radius $r$; the zero value corresponding to the surface center. The values of the wave number $\kappa$ obtained while shaking a pure water surface between 12 and $79 \mathrm{~Hz}$ are displayed in Fig. 3 .

The changes of the interface shape are characterized when the interface is progressively contaminated with palmitic acid, for an arbitrary frequency of $32 \mathrm{~Hz}$. Three surface concentrations are studied. For two of them, the wave slope envelope is displayed in Fig. 4. As expected the wave number and the damping coefficient increase progressively with the surface concentration.

Thanks to these measurements the evolution of the surface tension against concentration is determined with the re- lation (25) as shown in Fig. 5. This method is not a direct measurement of the surface tension but it is a non-intrusive method giving an absolute value of the surface tension, which stand for two non-negligible advantages. The dilational elasticity obtained from relation (26) is presented in Fig. 6.

\section{Conclusion}

A simple mathematical procedure ensures the determination of the elasticity modulus $\epsilon$ and the surface tension $\sigma$ from non-intrusive measurements carried out on a functionalized interface populated by nearly standing capillary waves. Such a rheology characterization have rarely been performed, except by Saylor et al. [9]. Important improvements concerning both the experimental setup and the dilational rheology identification are proposed so as to improve the accuracy of the method, particularly by scanning the interface around its center. A linearized form of the surface elevation is proposed in order to make easier the wave slope recognition, nevertheless an identification method could improve this recognition stage. The surface tension is calculated through the solvability condition for the coefficients $A$ and $B$ in the real and imaginary parts of the normal stress relation. Thanks to this relation, a minimization method is not more necessary to obtain $\sigma$ and $\epsilon$. More measurements have to be carried out to truly exhibit the abilities of the method, especially with the characterization of bio-molecules.

\section{References}

[1] J. Berthier, L. Davoust, PCT Intl. Appl. No. WO03/00920, French Patent No. EN0203690 (2000).

[2] E.H. Lucassen-Reynders, J. Lucassen, Adv. Colloid Interf. Sci. 2 (1969) 347.

[3] T. Vo-Dinh, B. Cullum, Biosensors and biochips: advances in biological and medical diagnostics, Fresenius J. Anal. Chem. 366 (2000) 6-7.

[4] D. Thomas, L. Blum, A. Girard-Egrot, Development of a fluid functionalized lipidic matrix applied to direct in situ polynucleotide detection, Biosens. Bioelectron. 20 (2005) 1539-1548.

[5] C. Barentin, C. Ybert, J.-M. Di Meglio, J.-F. Joanny, J. Fluid. Mech. 397 (1999) 331.

[6] D. Mobius, R. Miller (Eds.), Drops and Bubbles in Interfacial Research, Elsevier, 1998, p. 327.

[7] E.J. Bock, J. Colloid Interf. Sci. 1147 (1991) 422-432.

[8] J.A. Nicolas, J.M. Vega, A note on the effect of surface contamination in water wave damping, J. Fluid Mech. 410 (2000) 367-373.

[9] J.R. Saylor, A.J. Szeri, G.P. Fouks, Exp. Fluids 29 (2000) 509. 